\title{
Proyecto Francés y Educación: veinte años de esfuerzos para el mejoramiento de la enseñanza del francés en Costa Rica
}

\author{
María Gabriela Núñez Quesada
}

Universidad Nacional, Costa Rica

María Gabriela Vargas Murillo²

Universidad Nacional, Costa Rica

\section{RESUMEN}

La actividad de sistematización efectuada con los proyectos de la Escuela de Literatura y Ciencias del Lenguaje permitió trazar grandes líneas para el mejoramiento de la enseñanza del francés en Costa Rica. Además de un panorama histórico del nacimiento, desarrollo y evolución del proyecto, se of rece un resumen de los logros más relevantes y de las proyecciones hacia el futuro, que ya se marcan con claridad, así como un estado actual de la situación de los docentes de francés en nuestro país.

\section{RÉSUMÉ}

L'activité de systématisation mise en marche par projets de l'Ecole de Littérature et Sciences du Langage a permis au projet Français et Education de dresser les grandes lignes dans l'amélioration de l'enseignement du français langue étrangère au Costa Rica. En plus d'un panorama historique de la naissance, développement et évolution du projet, on vous présente un

1 Correo electrónico: gabynunez@latinmail.com.

2 Correo electrónico: literat@una.ac.cr. 
résumé des buts atteints, des projections vers le futur, marquées clairement dès maintenant, et un état actuel de la situation des enseignants de français dans notre pays.

Palabras clave: Francés, enseñanza.

Mots-clés : Français, enseignement.

\section{Los orígenes}

En 1984 y los profesores de la sección de Francés de la Escuela de Literatura y Ciencias del Lenguaje (ELCL) de la Universidad Nacional (UNA) emprendió una reflexión sobre la situación de la enseñanza del francés en Costa Rica que desembocó en la creación de un proyecto para su mejoramiento. A falta de contacto de los profesores graduados con posibilidades de formación continua, aunada a la cantidad de profesores no titulados que laboraban en zonas alejadas se sumaron las muestras de deficiencia en la enseñanza del francés en diversos niveles de la educación formal del país y la necesidad de modificar positivamente este proceso por medio de la proyección del quehacer de la Escuela y la retroalimentación con la realidad educativa. Entonces, el francés se impartía únicamente en la enseñanza secundaria. Ante ese panorama, en 1985 se fundó el Proyecto de Interacción con Profesores de Francés (PIPF). El equipo inicial quedó conformado por Julia María Gamboa, Alma Rosa Aguilar, María Gabriela Núñez Quesada y Virginia Vega. El apoyo de la Embajada de Francia y del personal de la ELCL en materia de extensión universitaria fueron claves para la puesta en marcha del proyecto. Hasta el presente, el acompañamiento continuo por parte de la cooperación francesa y las autoridades costarricenses relacionadas con la enseñanza en Costa Rica han permitido cristalizar las diferentes etapas que el proyecto ha atravesado durante su consolidación.

El proyecto se formuló con los siguientes objetivos: 
- Contribuir al mejoramiento de la enseñanza del francés en el país.

- Colaborar con una reestructuración de los programas vigentes -en la Universidad y en la educación secundaria- para adaptarlos a la realidad del estudiante costarricense y de sus intereses.

- Proponer una alternativa de desarrollo para la Unidad Académica que, centrada en la extensión, generase actividades novedosas en docencia e investigación, ligadas a las necesidades más urgentes de la realidad del país.

- $\quad$ Establecer contactos con el Ministerio de Educación Pública (MEP), la Universidad Estatal a Distancia (UNED), la Universidad de Costa Rica (UCR), la Oficina de Acción Lingüística de la Embajada de Francia y otras entidades interesadas en el quehacer para el mejoramiento del francés en nuestro país.

Los mismos objetivos que dieron origen al PIPF en 1984 conservan su vigencia a pesar de las variaciones históricas y los cambios en las corrientes educativas, ya que la problemática sobre la enseñanza del francés la han acentuado la formación y la actualización de los docentes, y las dificultades de comunicación e intercambio con un medio francohablante que les permita contextualizar sus labores docentes y favorecer el aprendizaje de sus estudiantes. Lo que empezó como una modesta iniciativa de actualización, ha abarcado otros campos de apoyo docente y de proyección universitaria, y para cada actividad tradicional se ha abierto paso una nueva oportunidad de crecimiento y proyección.

\section{Sector meta del proyecto}

Por su naturaleza, el proyecto actualmente cuenta con dos sectores meta: los maestros de primaria y los profesores de secundaria de francés de todo el país; pero estos sectores no son los únicos; también se benefician indirectamente otro sector: los estudiantes 
universitarios. El profesorado de francés son el sector meta principal, pero su efecto multiplicador en sus propios estudiantes es la principal fortaleza del proyecto. Por cada profesor que se actualiza o que recibe alguna capacitación, se beneficia un promedio de 400 estudiantes que están a su cargo en la institución de primaria o de secundaria donde labora. Así, sólo en el taller realizado en junio de 2006 en Heredia, al que asistieron 46 profesores, se favorecería al estudiantado; es decir, unas 18.400 personas.

Los destinatarios han intervenido siempre utilizando los medios más diversos: algunos han externado sus expectativas durante las visitas o en los talleres, porque prefieren un contacto con los extensionistas; otros se han comunicado por vía telefónica; algunos han enviado cartas o mensajes en las encuestas solicitando alguna ayuda en especial para su caso e incluso se ha incrementado el número de consultas por internet para recabar información (por ejemplo, la trascripción de artículos de la ley 7600 en sus dimensiones educativas e incluso dudas sobre aspectos técnicos de evaluación o prácticas de clase). Ha sido esa diversidad de necesidades la que ha impulsado la multiplicidad de opciones y respuestas.

En la actualidad, el sector meta original se ha cuadruplicado; entre algunos cambios acaecidos se encuentran algunos que han agravado el problema de la formación profesional de los docentes. A la vez, la inclusión del francés en la primaria ha planteado el problema de la articulación entre los niveles educativos, que no ha sido posible cristalizar. Por otra parte, otra inclusión del francés, esta vez en los primeros tres años lectivos de la enseñanza técnica, ha aumentado la necesidad de profesores de francés en zonas rurales y a la vez ha mostrado que los que se encuentran nombrados no cumplen en la mayoría de los casos con los requisitos necesarios para el puesto que desempeñan. A esta situación se une la dificultad de formación planteada por la disposición gubernamental de impartir doscientos días de clase, lo que ha bloqueado la formación continua y la capacitación para los profesores, ya que ni los directores de las instituciones 
ni los directores regionales conceden permisos para estos fines, ante el riesgo de una sanción. Esta contradicción entre la obligación del MEP de capacitar a sus docentes y su directriz de cumplir un número determinado de días lectivos, ha perjudicado no sólo a los profesores de francés, sino a los miles de estudiantes que dependen de ellos, ya que temas que necesitan ser tratados con urgencia, como las adecuaciones curriculares, no son objeto de interés real por parte del MEP.

\section{La conceptualización universitaria de la extensión y el proyecto Francés y Educación}

Al surgir como universidad necesaria, la Universidad Nacional ha concebido la extensión como factor de unión entre la docencia, la investigación y la acción social, como

una actividad básica del quehacer universitario. Constituye la tarea por medio de la cual la universidad se interrelaciona crítica y creadoramente- con la comunidad nacional. Proyecta a la sociedad, de la que la universidad forma parte, el producto del quehacer académico, a la vez que lo redimensiona y enriquece al percibir las auténticas y dinámicas necesidades de la sociedad. Con ello se persigue una formación integral tanto en la labor docente como estudiantil, una investigación comprendida con los cambios que reclama el desarrollo nacional ${ }^{3}$.

En la Escuela de Literatura y Ciencias del Lenguaje la extensión ha sido un medio para acercarse a las actividades pedagógicas de las instituciones escolares y para realimentar sus propias actividades docentes y de investigación. En la Memoria del XX aniversario se afirma: "Por un lado (la extensión) es el resultado natural de los proyectos de investigación y de las necesidades docentes...y por el

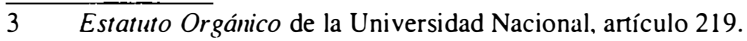


otro, un instrumento detector de necesidades que otras áreas deben tomar en cuenta para su resolución",4.

El profesor Ronald Miranda enuncia las cinco modalidades de la extensión en la Universidad $\mathrm{Nacional}^{5}$, presentes desde los inicios en el proyecto Francés y Educación:

- Comunitaria: porque a lo largo de los años el proyecto ha colaborado en todos los cambios educativos relacionados con la enseñanza del francés, respondiendo a las necesidades de la comunidad costarricense: las visitas a múltiples instituciones educativas de las más diversas regiones y modalidades han beneficiado a toda la comunidad nacional. Ejemplos son los talleres y asesoría en zonas alejadas del país, así como los diagnósticos realizados en diferentes oportunidades por medio de encuestas, que han identificado las necesidades de formación en las diversas realidades educativas de cada comunidad.

- Académica: por la gran cantidad de productos pedagógicos y lingüísticos que a lo largo de veinte años el proyecto ha desarrollado, algunos de ellos incluso publicados con sello editorial y otros divulgados en revistas o encuentros nacionales e internacionales.

- Cultural: por su vocación intrínseca y su naturaleza multicultural, el proyecto ha facilitado la integración y la armonía de la cultura francohablante y la identidad costarricense, potenciando así la identidad cultural y el respeto a la diferencia como lo prevén las orientaciones estratégicas de la extensión emanadas en 2003.

- De difusión y divulgación: por el esfuerzo de comunicación con los docentes de todas las modalidades y regiones del país, para entablar con ellos un diálogo académico que les haga llegar las propuestas pedagógicas elaboradas: emisiones de radio en Radio Nacional, boletines de información y página web en elaboración.

$4 \quad$ Memoria del XX aniversario (1994), Escuela de Literatura y Ciencias del Lenguaje.

5 Ronald Miranda. Actas del Encuentro de Extensión Universitaria (UNA. 2001). 
- De capacitación docente: la mayor inquietud del proyecto, cristalizada a través de un sinnúmero de talleres en 20 años, sobre los más variados temas: lingüística, expresión oral y escrita, evaluación, motivación, nuevos enfoques en la enseñanza del francés, explotación de textos auténticos, comprensión global de documentos, estrategias de aprendizaje, inteligencias múltiples, nuevas tecnologías en la clase de lengua extranjera, elaboración de material didáctico, literatura, galicismos, cultura, derechos humanos, ecología, la familia.

En octubre de 1996 en una mesa redonda en la Universidad Nacional, Gil Ramón González, del Ministerio de Educación Superior de Cuba, manifiestó: "La extensión tenemos que verla como una función, como un proceso inmerso en los demás procesos de la universidad"6. Durante esa actividad, el entonces rector Jorge Mora Alfaro, expresó que "el conocimiento no se crea de manera exclusiva en la universidad sino que se genera mediante el cambio permanente, el contacto, la relación directa con los diferentes procesos de desarrollo y los distintos actores sociales"7. En este marco, la sección de Francés ha sido pionera de los proyectos de extensión universitaria: PROFE es el proyecto más antiguo y constante de la ELCL. En el ámbito nacional, ninguna otra universidad, pública o privada, ha incursionado en esta modalidad de extensión, tan diversificada e ininterrumpidamente. Cabe destacar que casi la totalidad de los miembros en propiedad y de los profesores pensionados de la sección han formado parte del equipo de extensionistas en algún momento, de manera que la proyección hacia la comunidad nacional ha sido continua.

\section{El quehacer de dos décadas de extensión}

El equipo ha mantenido colaboración con entidades relacionadas con la Enseñanza del Francés, como la Asociación Costarricense

\footnotetext{
$6 \quad$ Transcripción de las intervenciones de la Mesa Redonda: "Papel de la extensión en los procesos de cambio de nuestras sociedades" (UNA, 1996).

7

Idem.
} 
de Profesores de Francés (ACOPROF), el Ministerio de Educación Pública (MEP), la Universidad de Costa Rica, la Embajada de Canadá y la Oficina Pedagógica de la Embajada de Francia.

El proyecto empezó en 1985, al conformarse el primer equipo de extensionistas, desde ese momento se logró contacto con el MEP. Se planearon giras a zonas rurales como Guanacaste, Limón, Puntarenas y la zona sur, para aplicar un cuestionario que permitiera luego un análisis de datos sobre los profesores de francés de secundaria y sobre sus experiencias, necesidades y expectativas, para detectar las futuras áreas de acción del proyecto. Se corroboró la inquietud de los docentes con respecto a su asesoramiento de parte de los organismos correspondientes, así como sus expectativas por un mejoramiento profesional. Al preguntar cuáles áreas consideraban más necesarias para un seminario de actualización, un 29\% deseaba expresión escrita y oral, un $21 \%$ estaba interesado en metodología, $17 \%$ en civilización (i.e. cultura francesa), $14 \%$ en fonética, $12 \%$ en gramática, $5 \%$ literatura y $3 \%$ lingüística. En ese tiempo se establecieron como zonas prioritarias Limón y la región Sur del país. Directores, asesores y autoridades externaron además que dificultades de tipo administrativo impedían muchas veces una labor eficiente, por lo cual se estableció un contacto con ellos gracias a un archivo de nombres y direcciones preparado con material de los archivos del departamento de personal y que facilitaría el acercamiento hacia los profesores. Ese primer año de trabajo contó con la incorporación de Wolf Hollerbach, profesor invitado de la Universidad de Alaska.

En el año siguiente se publicó el primer boletín bimensual y se realizó un curso de capacitación en la zona de Limón que comprendió cinco giras con diferentes temas de interés, con una duración de seis meses $^{8}$. En 1987 se completó la actualización del diagnóstico, por

$8 \quad$ Para ver más detalles sobre la serie de talleres de capacitación impartidos en Limón, puede revisarse el documento correspondiente a la recopilación hecha por las extensionistas responsables. Se escoge Limón debido a que presenta características especiales: un alto empeño de los docentes de la región en participar y la posibilidad de un local fijo para impartir las capacitaciones (sala de profesores del Liceo Diurno). 
medio de la aplicación de un cuestionario. Se realizó también la tabulación y el análisis de los cuestionarios ${ }^{9}$. Para el mes de mayo se tuvo una primera sesión de trabajo con docentes de Alajuela. Posteriormente, se realizó un taller en la Sede Central donde asistieron profesores de diversas zonas del país, se promovieron la expresión oral y el mejoramiento metodológico. Durante el resto del año, se facilitó material didáctico a aquellos profesores que se desplazaron a la sede de Heredia y a Alajuela.

En 1988 se firmó una Carta de Entendimiento entre la UNA y la Embajada de Francia para consolidar los esfuerzos realizados en aras del mejoramiento de la enseñanza del francés; un gran logro ya que con esto se oficializó el apoyo que la Oficina Pedagógica había brindado en cuanto a materiales, comunicación con los profesores, bibliografía, preparación del profesional del equipo de extensionistas, entre otros.

La reimplantación de los exámenes de Bachillerato en el sistema educativo conllevó cambios en cuanto a la enseñanza de las lenguas, con lo que se hizo evidente cierta anarquía en la escogencia del método empleado en su aprendizaje. En el caso del francés, se detectó una coexistencia de diversos métodos de diferente naturaleza, algunos estructuralistas y con principios comunicativos, los cuales multiplicaban los objetivos en forma poco armoniosa. En ese momento, se empleaban cuatro manuales: Mise au point, Orange, La France en Direct (1987) y Sans Frontières ${ }^{10}$. Este contexto permitió la realización de varios talleres (en Limón y en San Ramón se of recieron talleres de lectura global; también se organizó un taller de evaluación y otro de comprensión de lectura para la región de Alajuela) y seminarios (sobre lectura global) que lograron reunir a muchos de profesores.

En el Seminario regional de COPALC-FIPF se constató la importancia de unificar los objetivos de enseñanza-aprendizaje del

$9 \quad$ Es importante hacer notar que el procesamiento de datos en aquellos momentos era lento y dificultoso. por lo que las extensionistas solicitaron colaboración de especialistas en estadística para realizar este trabajo.

10 Guy Capelle y otros, La France en Direct (Alcobendas: Sociedad General Española de Librería, 1987). 
francés y se lanzó entonces la propuesta de reforma al plan de Bachillerato en la Enseñanza del Francés de la UNA para actualizar la of erta académica.

El proyecto empezó sus labores de indagación bibliográfica sobre técnicas de comprensión de lectura y evaluación y participó en otros seminarios, como el de FIPF-COPALC (Comisión para América Latina y Centroamérica de la Federación Internacional de Profesores de Francés) y un seminario de lectura con Sophie Moirand.

Se elaboró, además, una propuesta para la creación de cursos libres con profesores de francés en servicio en la ELCL. Durante 1989 se ratificó el primer acuerdo con una reformulación de la Carta de Entendimiento y se firmó un Convenio de Cooperación entre la UNA y la Embajada de Francia que formalizó la colaboración entre la Oficina de Acción Lingüística y el equipo de extensionistas del Proyecto de Interacción con Profesores de Francés (PIPF). Esta ratificación pretendía unir los esfuerzos de ambas instituciones y ofrecer al profesorado los materiales y apoyo pedagógico necesarios. Asimismo, esta colaboración garantizaría una comunicación permanente y efectiva con los profesores de regiones alejadas. Se formuló un curso de traductología y un taller de motivación para profesores. Los cuatro extensionistas también participaron en formaciones y seminarios, como el seminario de Evaluación impartido por el especialista francés J. C. Mothe,

Se realizó la presentación de una ponencia en el seminario Regional de la COPALC-FIPF, así como la preparación de una ponencia para SEDIFRALE en Brasil. El proyecto colaboró con la puesta en práctica del proyecto experimental costarricense La Fenêtre como método para la enseñanza secundaria ${ }^{11}$. Ese mismo año, a raíz de la implantación del Bachillerato en Secundaria, el equipo se abocó a redacción de ejercicios de comprensión de lectura (un cuaderno de ejercicios, folletos y documentos sobre metodología y evaluación) y

11 Ligia Salas. La Fenêtre (San José: Librería Francesa, 1992). 
la visita a diversas regiones. Además, se colaboró activamente con la Asesoría Nacional de Francés en la preparación de los profesores de secundaria para este examen, que había sido eliminado desde 1974.

Para formación del prof esorado en servicio se of recieron en San José dos talleres, uno de evaluación y otro sobre la motivación para la clase de francés. Para la proyección a las regiones rurales, se impartieron cursos taller sobre evaluación y metodología en Limón y en Alajuela. Los extensionistas se abocaron también a su autoformación y participaron en programas de especialización en el extranjero. El equipo se dedicó a la tarea de la formación de profesores en servicio mediante cinco cursos cortos. Se elaboraron tres tipos de materiales, uno para cada nivel de sétimo a noveno, con objetivos, fichas pedagógicas, selección de textos y ejecución pedagógica. Los extensionistas iniciaron una nueva modalidad de consultoría, en la cual los profesores interesados se apersonaban a la Escuela de Literatura a plantear sus dudas y recibir atención personalizada por parte de los extensionistas.

La labor de estos años estuvo marcada por mucha producción de materiales. Se elaboraron artículos en revistas internacionales especializadas sobre la gramática y el aprendizaje del francés. Se asistió con una ponencia sobre la gramática a SEDIFRALE VIII, en Santiago de Chile. Sale a la luz Gool, lecturas en francés de textos deportivos y se concretó la publicación de la guía pedagógica y del cuaderno de lectura Le français au fond des yeux. Y se concibe la primera versión del cuaderno de lectura sobre ecología Echo logique.

A partir de 1994 se organizó en el Ministerio de Educación Pública un programa para la enseñanza de lenguas extranjeras en primaria (PROLED), actualmente Oficina de Lenguas Extranjeras (OLE) que ha permitido una apertura al francés para niños en las escuelas públicas; hoy día se enseña en treinta y dos escuelas de San José, Heredia, Guanacaste y Cartago. A partir de ese momento el proyecto se amplió; su sector beneficiario se extendió a todos los docentes de primaria que imparten francés, actualmente son unos cien maestros. 
Durante este periodo se trabajó también en secundaria, que había renovado sus programas de estudio, con ayuda de una comisión en la que el PIPF participó; y se concretó el Plan Regional de Actualización para Profesores de Alajuela, con diez sesiones de cuarenta horas con diversos talleres sobre planeamiento, evaluación, gramática y literatura. Durante tres jornadas pedagógicas se realizó la presentación de material de Le Français au fond des yeux. Además, hubo una jornada pedagógica en el Liceo de Esparza, para el análisis de los procedimientos pedagógicos en el trabajo con los métodos oficiales de secundaria: Le français chez nous y Bonne route. En coordinación con la Asesoría Nacional de Francés, dio asesoramiento a la subregión de San José con el tema de evaluación de lenguas extranjeras, específicamente en las cuatro habilidades. Con el fin de cubrir más regiones, se visitó un total de ocho colegios (Nicoya, León Cortés, San Carlos, Quepos, Cariari, Juan Viñas, Naranjo) que. gestionaron asesoramientos particulares para sus necesidades.

Durante este tiempo, el equipo coordinó con la Dirección Regional de Cartago la puesta en marcha del Plan Regional de Actualización de Profesores con una duración de cuarenta horas y un asesoramiento con la Dirección Regional de San José, con temas como la gramática textual, la evaluación, la elaboración de pruebas, la narración y la expresión oral y escrita.

Se planteó una ampliación y revisión del cuaderno de lectura sobre ecología y digitación de fichas. Dentro de la producción de material, se revisa La grammaire autrement, folleto para la enseñanza de la gramática a nivel avanzado. En cuanto a la labor con los maestros de primaria, se realizó del 13 al 23 de febrero un asesoramiento de cuarenta horas con diseño de materiales, evaluación de competencias, estrategias metodológicas para la comprensión y producción, actividades lúdicas y creatividad.

Se culminó con el libro Echo Logique, con una última revisión de diagramación, ilustraciones y la edición, así como la organización 
de actividades de difusión del mismo ${ }^{12}$. También se completó una última revisión de La grammaire autrement. Se elaboraron dos unidades didácticas para Educación Diversificada: "La famille" y "Les droits de l'homme", y se impartió un seminario de actualización en Heredia para la puesta en marcha de los nuevos programas del MEP y del nuevo método Fréquence Jeunes para secundaria ${ }^{13}$.

Se continuó con actividades de actualización en diversas regiones: San José, Puntarenas, Guanacaste, Heredia y Limón, con la colaboración de las Direcciones Regionales del MEP. Asimismo, el equipo participó con otras instituciones (el MEP, la UCR, el IIMEC, la Embajada de Francia y la ACOPROF), en la elaboración de una edición especial para Costa Rica del método Fréquence Jeune 2 y de su guía pedagógica. Este fue el método oficial de francés para décimo y undécimo años.

El proyecto presentó una ponencia en el Congreso Iberoamericano de Extensión y el coordinador realizó una pasantía en el CAVILAM (Centro Audiovisual de Lenguas Modernas), con el auspicio del Bureau d'Action Linguistique (BAL), de la Embajada de Francia. A raíz de esta participación en el Cavilam, Róger Retana inició las producciones de las emisiones de radio. Con ayuda de los cooperantes francohablantes de Shawinigan Internacional (Canada) se grabaron cuarenta emisiones (cada una de quince minutos, para un total de más de diez horas continuas) de Fréquence ADO. Cada emisión contó con un guión y con la respectiva ficha pedagógica que permitirá compilarlos y ponerlos a disposición de los profesores.

El equipo continuó colaborando en la elaboración de la guía pedagógica para Fréquence Jeunes 2; se prosiguió la recopilación de material auténtico, la elaboración de fichas, el tratamiento pedagógico de los materiales, la corrección de estilo y revisión de maquetas.

$\overline{12}$ Actualmente, este libro es el texto del curso Análisis de Textos, de las carreras que ofrece la sección de Francés.

13 Guy Capelle y otros. Fréquence Jeunes (París: Hachette Education, 1994). 
La década de 2000 se inicia con un nuevo nombre para el proyecto, dada la diversificación de acciones y del sector destinatario: el Proyecto Francés y Educación, (PROFE); su equipo participó con tres ponencias en el Congreso Regional Centroamericano en Granada, Nicaragua sobre la utilización de la radio en la clase de francés (Róger Retana), la enseñanza del francés para niños (María Gabriela Núñez) y la elaboración y puesta en marcha en Costa Rica de Fréquence Jeunes 2. Se completan cincuenta emisiones más del programa Fréquence $A D O$, con un total de 12,5 horas de grabación, siempre contando con la colaboración de los cooperantes extranjeros. Cada emisión de 15 minutos supuso diez horas de trabajo, entre la búsqueda del material y los contactos, realización, montaje, edición y elaboración de la ficha pedagógica. Las grabaciones se realizaron en diversos lugares, incluso fuera de Costa Rica (Francia, Canadá, Estados Unidos, Guatemala, Nicaragua, Alemania), con el acompañamiento de fichas pedagógicas y guiones para cada una.

El equipo de extensionistas participó en varias actividades internacionales: el Congreso Nacional de Profesores de Francés, el Encuentro Centroamericano de Profesores de universidades centroamericanas, un taller en Guatemala sobre actividades orales en clase, animación de talleres en la región centroamericana en Panamá (de 40 horas), Costa Rica (8 horas) y Honduras sobre la enseñanza del Francés Lengua Extranjera a niños y el Seminario Regional de Profesores de Francés, en Tela, Honduras.

En 2001 PROFE realizó una importante actividad especial: una reunión en 2001, con cien profesores de francés procedentes de todas las provincias del país, para hacerles entrega de una selección con las doce mejores emisiones producidas por Fréquence $A D O$, gracias a un esfuerzo conjunto de la UNA, el CCCAC y el Colegio de Licenciados y Profesores. A este encuentro asistieron las más altas autoridades de las instituciones involucradas.

Los extensionistas se autoformaron en el campo de las nuevas tecnologías y recibieron capacitación en el uso de la computadora y la 
creación de sitios web, además de su participación en tres congresos durante el año: el Congreso Turismo y Cultura, en Granada, Nicaragua, de donde surge la iniciativa de crear una red centroamericana para unir esfuerzos para el desarrollo en la región del francés con objetivos específicos (RECAFTUR), en el caso concreto de la formación de los profesionales de turismo.

Se consiguió la difusión regular de las emisiones de Fréquence Ado en Radio Nacional. Siempre en estrecha colaboración con los entes involucrados en la enseñanza del Francés en Costa Rica, en unión de cuales se completó una serie de reuniones de coordinación, PROFE realizó un total de doce talleres a lo largo del año, sobre temas diversos: el vídeo y la radio en clase, cultura, gestos y estereotipos, el método "Extra", actividades orales en clase de creatividad, la B.D., etc. Ese mismo año tuvo ocasión la puesta en marcha del proyecto RECAFTUR, que permitió a la UNA representar de manera exclusiva al país en un grupo selecto de profesionales centroamericanos, seleccionados por el Agregado de Cooperación Educativa de ese entonces, Patrick Dahlet, para realizar la adaptación del método "Vacances cubaines" para el aprendizaje del francés de turismo.

Con respecto a la formación continua con los profesores, se of recieron nueve talleres en coordinación con el CCCAC de la Embajada de Francia, y con el apoyo del Colegio de Licenciados y Profesores. Los temas fueron: las actividades lúdicas, los aspectos culturales en clase, la expresión oral, el video, la canción y la radio.

Los extensionistas fortalecieron su autoformación y participaron en el XII Congreso Internacional de SEDIFRALES, celebrado en Lima, Perú, con cuatro ponencias acerca de la radio, la adaptación de "Vacances cubaines" realizada por el equipo de la UNA, la enseñanza precoz en la oferta universitaria y la vida asociativa (de los profesores) en Costa Rica.

En 2004 se realizó una primera actualización de los datos de los profesores en el ámbito nacional. En coordinación con la División de Control de Calidad de MEP, se recabó información de todas las 
regiones a nivel nacional para actualizar las coordenadas de la gran mayoría de los colegas. Gracias a esto se inician los contactos mediante correo electrónico, lo que facilitó el envío de los dos primeros boletines bimensuales que se retomaron después de varios años de inactividad. Para diciembre de 2005 se contaba con 125 contactos (<proyectoprofe@hotmail.com>) y se habían atendido varias consultas, dando inicio así a la preparación de la página web del proyecto. Se realizaron siete talleres en Heredia, San José, Alajuela, San Carlos y se contó con la colaboración de especialistas canadienses, cubanos, franceses y costarricenses. Con la coordinación con la Asesoría Nacional de Francés, se aplicó una encuesta a los profesores de primaria para procesarla y ver las necesidades de formación de dicho público. Tres extensionistas participaron en el II Congreso Centroamericano de Profesores de Francés, con ponencias acerca de la radio en la clase, la enseñanza de la literatura a niños y adolescentes y la presentación del plan de estudios de la UNA de formación para docentes de francés para primaria, el cual fue planteado en el seno del proyecto y que se cristalizaría para 2005.

La actualización de los datos del diagnóstico nacional se ha fortalecido en los últimos años. A partir de una muestra de los profesores de secundaria del país se realizó un estudio acerca de la situación de los docentes de francés en Costa Rica. El informe correspondiente al diagnóstico nacional comprendió todas las encuestas aplicadas a los 177 profesores que conformaron la muestra, los resúmenes por región y sus respectivos gráficos, así como un análisis descriptivo de los resultados. En el informe se consignan las características de la muestra y su distribución en las regiones educativas del país, para analizar los datos obtenidos en dos niveles: por una parte se revisaron los resultados relacionados con aspectos académicos y laborales; por otra, se identifican las preferencias de formación continua que los docentes expresaron. Con respecto al nivel de formación universitaria de los profesores de francés, su condición laboral actual como interinos o propietarios, un cuadro acerca de los resultados en las 
pruebas nacionales de acreditación de francés, categoría dada por el MEP en relación con la condición de trabajo, el interés mostrado por la formación continua y las opciones de escogencia, temas de preferencia para recibir talleres o seminarios.

Resumir estos cuatro lustros de trabajo en etapas requiere cierta flexibilidad ya que a lo interno del proyecto, las nuevas épocas han estado enraizadas en experiencias y resultados anteriores, que han lanzado hacia nuevos derroteros las orientaciones de la labor de extensión. La primera etapa permitió al PIPF sumergirse en la realidad nacional y educativa de ese momento histórico. Durante los primeros dos años del proyecto (1985-1986) se intensificó la labor de diagnóstico para establecer, primordialmente, estrategias de auxilio inmediato para los profesores en servicio y, a la vez, estrategias para el trabajo de formación continua que se emprendió de manera regular y que continúa al presente: jornadas pedagógicas, talleres, cursos periódicos, encuentros con profesores, pasantías coordinadas con la Oficina pedagógica de la Embajada de Francia, eventos en coordinación con el Colegio de Licenciados y Profesores, formaciones continuas e iniciales en cooperación el Ministerio de Educación, colaboración con ACOPROF en actividades pedagógicas.

$\mathrm{Al}$ establecimiento de las prioridades por atender, continúa una etapa de consolidación con entes colaboradores del proyecto: por una parte, se concretan planes de actualización en coordinación con el MEP y con otras instancias, a la vez que se acompaña a los profesores de francés en la transición hacia los exámenes de bachillerato, elaborando material pedagógico y realizando talleres de apoyo en comprensión escrita; por otra, se establecen acuerdos diplomáticos con la oficina pedagógica, que en ese momento era la Oficina de Acción Lingüística (BAL) para permitir mayor efectividad en las acciones conjuntas .

Hay una tercera etapa (1990-1998), de gran producción de material didáctico, marcada por los cambios en los programas de estudio y en las teorías del aprendizaje de lenguas extranjeras en 
ese momento. Este es el período donde se concretan varios productos, algunos publicados incluso bajo sello editorial y en revistas internacionales ${ }^{14}$.

Una etapa con las "tecnologías de la comunicación" se gesta a partir de 1999 con la difusión de las emisiones radiofónicas. Se alcanza una mayor cobertura, porque los beneficiarios antes indirectos se convierten en directos: los estudiantes pueden disfrutar personalmente de la labor del proyecto en su colegio o en su casa al sintonizar la programación de Fréquence ADO en Radio Nacional. Esta etapa lleva al empleo de recursos de comunicación actuales (el fax, por ejemplo) para establecer una efectiva comunicación con los profesores e informarlos de las actividades de capacitación.

La última etapa del proyecto podría fijarse en 2004, con el inicio de las proyecciones para la labor en Internet y el enfoque "cibernético" de la formación continua y de la consultoría pedagógica, por medio del correo electrónico, así como la producción de material informatizado y digitalizado que estará a disposición de gran número de público en un futuro cercano ${ }^{15}$.

En el paso por las diferentes etapas no se abandonó la labor de las anteriores, sino que se ha expandido el campo de acción, integrando al volumen de trabajo normal una mayor cantidad de tareas, lo que ha desembocado en la imperiosa necesidad de diversificar el proyecto y plantearlo como un conjunto de proyectos interrelacionados, pero con autonomía. Para 2006, además de PROFE, el programa Fréquence $A D O$ se ha constituido en proyecto independiente, bajo la dirección de Virginia Boza Araya y para 2007 se emprenderá un nuevo proyecto que diseñará un modelo pedagógico para la enseñanza del francés específicamente a niños desde preescolar hasta sexto grado de enseñanza primaria.

14 Resumido a partir de informes parciales de labores del PIPF de 1985 al 2000.

15 Resumido a partir de informes parciales de PROFE (2000-2004). Se podrá consultar este material en < $w^{\prime} w$ w.una.ac.cr/litertura $>$ : incluye entre otras secciones. la historia del proyecto desde sus inicios y la sección dedicada a las emisiones de radio de Fréquence $A D O$ de 1999 y 2000 . El sitio fue desarrollado gracias al apoyo de la ELCL. en la persona de la asistente en informática de los laboratorios. Joseth Trigueros. quien puso en marcha el diseño realizado por las extensionistas. 


\section{El diagnóstico de la situación del profesorado de francés en Costa Rica}

El proyecto Francés y Educación ha sido el gestor de iniciativas de diversa naturaleza a lo largo de sus veinte años de vigencia. El diagnóstico de necesidades de los profesores en servicio en el país, realizado hace dos décadas, fue iniciada en 2004 y actualizada en 2005 , para poner al día los resultados y elaborar proyecciones concretas y efectivas para las próximas temporadas.

La actualización del diagnóstico se realizó con la cooperación de la Asesoría Nacional de Francés, la Asesoría de Macroevaluación de la División de Control de Calidad del MEP y la ACOPROF. En 2004 arrancó un proceso de coordinación con la Asesora Nacional de Francés, que permitió el establecer el instrumento con el cual se recabó la información. Se utilizó una muestra representativa de todos los profesores del país que pudo ser analizada por las Asesoras Nacionales de Macroevaluación en sus giras de 2005. La muestra estuvo conformada por los 177 profesores asistentes a las sesiones de capacitación, los cuales tenían a cargo sus diferentes niveles en la educación secundaria. Entre los aspectos que se estudiaron se encuentran: nivel académico y actual condición laboral, estabilidad y categoría laboral, modalidad de estudio o de formación de preferencia, nivel de conocimiento acerca de la existencia del proyecto Francés y Educación y preferencias en los temas de capacitación.

\section{La situación en la enseñanza primaria}

En 1994 se puso en marcha enseñar lenguas extranjeras a los niños escolares del país, gracias a la Oficina de Lenguas Extranjeras (OLE), del MEP. Este proyecto contempla la enseñanza de una lengua extranjera a los niños de la enseñanza primaria. De esta forma, las escuelas pueden impartir francés o inglés como lengua extranjera. Hoy, este proyecto abarca una mayoría de escuelas que of recen el 
inglés y 55 escuelas en las regiones de San José, Heredia, Cartago y una escuela en Guanacaste que of recen el francés. Existen dos escuelas bilingües donde el francés se enseña no sólo como lengua extranjera, sino que es la lengua en la que se enseñan las materias básicas.

La encuesta realizada a finales de 2004 incluyó 22 docentes de primaria de diversas escuelas, convocados por la Asesora Nacional de Francés a un asesoramiento en noviembre. Según los datos recabados, estos docentes imparten diversos grados, de manera que todos los niveles estaban bien representados. El panorama de este sector es más reducido, ya que a pesar de las diversas regiones de procedencia, la escasa cobertura con que se cuenta actualmente OLE para francés no permite una proyección completa de las regiones urbanas y rurales del país, sino sólo de aquellas que cuentan con escuelas que ofrecen francés.

Los grupos profesionales son variados. Algunos de los docentes tienen una calificación como profesores de secundaria (once de ellos), con incluso graduados universitarios (seis bachilleres y dos licenciados) y el resto ya ha obtenido calificaciones como docente de primaria. Sin embargo, solo uno de ellos afirma tener propiedad. Vale destacar que entonces un $95 \%$ de los entrevistados no tiene estabilidad laboral, a pesar de que el $36 \%$ posee un título universitario.

El conocimiento de la lengua lo han obtenido por diversos medios, en ocasiones combinados, pero predomina el estudio, sea en Costa Rica o del exterior, con un 90\%. Los encuestados realizan o realizaron sus estudios principalmente en la Universidad de Costa Rica y en el convenio UCR-UNED.

La mitad de los entrevistados prefiere estudiar mediante la modalidad presencial y un $45 \%$ según la modalidad a distancia, con preferencia marcada (un 59\%) por los horarios de viernes por la noche y todo el día sábado. Según expresaron los encuestados, si la UNA ofreciera este horario para la formación universitaria, se matricularía un $40 \%$ de ellos. A todos les interesó recibir los boletines pedagógicos ofrecidos por PROFE, pero un $72 \%$ desconocía la existencia del 
proyecto. Pese al escaso interés en la educación virtual, un $63 \%$ desea recibir información principalmente a través del correo electrónico. Esta paradójica realidad debe estimular esfuerzos por hacer llegar la tecnología a los profesores y por estimularlos en el uso de ésta, tanto para mejorar su desempeño profesional como para motivar a sus estudiantes y ligarse al mundo francófono.

\section{La situación en la educación secundaria}

Hoy, se calcula en unos 700 el número de profesores que en el país enseñan el francés en la secundaria. La muestra de este estudio abarca 177 docentes de las regiones educativas del país, lo cual representa aproximadamente un 25\%. La distribución de los encuestados se desglosa de la siguiente manera según región de procedencia: San José (sector central), 25; Desamparados,19 ; Puriscal, 2; San Isidro de Pérez Zeledón, 12; Heredia centro, 12; Heredia (alrededores), 7; Alajuela,9; San Carlos, 22; Cartago, 3; Turrialba, 2; Puntarenas, 9; Aguirre, 2; Limón, 15; Guápiles, 5; Guanacaste, 27 y Cañas, 6.

Las personas consultadas laboran en instituciones públicas y privadas y tienen a su cargo todos los niveles de la secundaria. La muestra se escogió por conveniencia y abarcó la totalidad de los docentes asistentes a las reuniones de información y capacitación sobre las Pruebas Nacionales que se realizaron entre abril y mayo de 2005, a cargo de las Asesoras Nacionales de Macroevaluación. Durante esas sesiones, los profesores recibieron la ponderación de los objetivos del temario que regirá las pruebas en el año, así como los resultados y estadísticas del año anterior, además, consejos, informaciones y guía para el mejor desempeño de sus estudiantes en el aula y en el rendimiento de las Pruebas. Los profesores convocados tenían a su cargo los niveles de $9^{\circ}$ y de $11^{\circ}$ años, pero, en razón de la cantidad de lecciones de francés por grupo, todos tienen, además, a su cargo al menos otro nivel y en ocasiones se encargan de los cinco niveles en instituciones muy pequeñas; el grado de representatividad es palpable. 
El primer aspecto por considerar es el nivel académico y la actual condición laboral de los docentes encuestados. De los 177 docentes entrevistados, un $97 \%$ afirma haber realizado algún tipo de estudio universitario o bajo la modalidad de la Alianza Francesa, pero no todos ellos completaron su formación hasta obtener un título. Una cantidad importante, 87 de ellos, continúa estudiando, principalmente con la opción of recida por el convenio de la UCR-UNED, que tiene tutorías únicamente los sábados y les permite combinar su trabajo con el estudio. De esta manera, el $49 \%$ recibe algún tipo de formación, sea porque busca un título o al menos superar la categoría de aspirante.

Las cabeceras de las provincias del Área Metropolitana y de los sectores predominantemente urbanos evidencian un alto porcentaje de graduados universitarios que ejercen la docencia. Los profesores con MT-4 o MT-5 e incluso MT-6 llegan al 76\% en el sector central de San José; $84 \%$ en Desamparados, $67 \%$ en el centro de Cartago, $75 \%$ en el centro de Heredia y $71 \%$ en los alrededores; baja en los centros de provincias del área rural como Alajuela, con 33\% y Puntarenas, con un 44\%; en ocasiones contrastan dramáticamente, como en el caso de Guanacaste, con un porcentaje de graduados de apenas un $14 \%$, y con un panorama desolador en Aguirre y en Turrialba entre cuyos entrevistados no hay graduados; en Limón tampoco hay graduados, con un $33 \%$ de aspirantes; en Cañas, de seis profesores asistentes, no hay un solo graduado y por el contrario la categoría aspirante ocupa un $67 \%$ de la clasificación; finalmente en San Carlos, donde el 0,9\% es personal graduado y los aspirantes conforman un $68 \%$ del total de docentes.

Junto a esta situación en cuanto a la formación universitaria completa está el panorama de estabilidad y categoría laboral en las diferentes regiones. Aquí la relación se modifica de manera preocupante, porque si la obtención de la propiedad podría ser considerada como incentivo para terminar los estudios, no necesariamente se da en la realidad. En algunos sectores urbanos se da estabilidad laboral, como en el caso del centro de Heredia, donde el $83 \%$ posee un puesto 
en propiedad y tiene título universitario; en otros sectores, esta estabilidad también se presenta, aunque la categoría asignada no sea alta, como el caso de Desamparados donde un $89 \%$ de los docentes está en propiedad, aunque de ellos hay un $17 \%$ con categoría MT-1 o MT2. En Puntarenas hay un $55 \%$ en propiedad, del cual un $11 \%$ no es titulado. Como casos especiales, la capital of rece un $40 \%$ de interinazgo, incluidos en este porcentaje varios graduados universitarios. El sector de Puriscal presenta un 50\% de interinazgo, a pesar de que el $100 \%$ es personal graduado.

Hay otros sectores donde, por el contrario, la falta de graduados no impide que los docentes hayan obtenido propiedad con categorías muy bajas, como los casos de Turrialba, Cañas, Guanacaste, Aguirre y en especial Pérez Zeledón, donde hay un $41 \%$ de propietarios, a pesar de que sólo el $16 \%$ tiene título universitario y el $25 \%$ restante apenas MT-1. En Guápiles hay un $40 \%$ de propietarios, pero sin titulación, con MT-2. Limón y San Carlos presentan un panorama inverso, con una gran mayoría interina ( $77 \%$ en el caso de San Carlos y $86 \%$ en el caso de Limón), aunque todos ellos con categoría de MT-1 o ASP, que muestra que el $75 \%$ de los docentes tiene escasa formación universitaria apropiada para el trabajo que desempeñan. El caso de Alajuela es interesante: hay un $67 \%$ de docentes en propiedad, aun cuando hay aspirantes en ese grupo, lo cual pone en evidencia la disparidad al contrastarse con sectores como Cartago, Heredia o Puriscal, donde se da el caso de graduados con MT-4 pero interinos. No hay una relación equilibrada entre la obtención de propiedad y el grado universitario que se posea. Se sabe que todos aquellos profesores con baja categoría, que poseen propiedad, generalmente tienen varios años laborando y no hay en sus sectores graduados que cumplan los requisitos de los concursos, de manera que les han sido otorgados por inopia. Por una parte, esta posibilidad de un trabajo fijo les ha permitido una tranquilidad económica siempre deseable desde el punto de vista emocional del docente, pero se pone en evidencia que estas mismas personas, ya con su propiedad, no expresan interés real por la formación continua 
y menos aún por finalizar sus estudios en alguna de las opciones existentes.

Por otra parte, se consultó sobre la modalidad de estudio o de formación preferida en caso de recibir algún tipo de capacitación. Los resultados contradictorios en relación con la experiencia del proyecto en los últimos años plantean un complejo problema; por una parte, la preferencia por la formación presencial es marcada: 125 (el 70\%); pero el proyecto constata que es difícil lograr una convocatoria efectiva de profesores a formación continua, a pesar de numerosos intentos de comunicación y del carácter gratuito de los talleres. Se preguntó a algunos de los encuestados lo que para ellos significa presencial; aquellos que viven en zona rural esperarían que la Universidad o el MEP lleguen hasta ellos y no en desplazarse por capacitación a la capital, como han tenido que hacerlo quienes siguen formación a distancia. Esta opción está ciertamente contemplada en las actividades del proyecto, pero no con la frecuencia deseada por los encuestados, quienes manifiestan interés porque fueran abiertos grupos de carrera en las sedes universitarias de zona rural. Según se ha comprobado a lo largo de veinte años, los docentes esperan una capacitación dentro de su horario laboral y no es de su principal interés el presentarse en sus horas libres para recibir talleres. De esta manera, cuando los talleres han sido convocados por el MEP, con permiso para ausentarse un día del colegio, la mayoría de los docentes se presenta sin que medie una convocatoria por parte del proyecto. Por el contrario, cuando la actividad ha sido organizada por PROFE, con asistencia fuera de horas laborales, ésta ha resultado inferior, a pesar de comunicaciones, correos, fax o llamadas telefónicas de invitación.

En segundo lugar de preferencia viene la formación a distancia, que muchos de ellos siguen actualmente y les permite trabajar al mismo tiempo. Esta opción sería aceptada por un total de 45 personas, un $25 \%$. Por último, con poquísimo eco, la formación virtual, con apenas 15 interesados, para un $0,8 \%$. Por otra parte, al consultar si se matricularían en talleres virtuales of recidos por la UNA, se obtiene un 
total de 116 interesados, es decir un 65\%. Muchos que descartan la opción virtual afirman "no tener internet o no tener computadora", lo cual pone en evidencia un asunto más profundo: luce contradictorio el rechazo a la formación virtual y al mismo tiempo el interés en ella. Al contrastar las informaciones, se verificó que la mayoría de los profesores con muchos años de servicios sostienen su preferencia por talleres virtuales, por el contacto con otras personas y desconfían de la internet "por su despersonalización"; sin embargo, hemos confirmado que estos mismos profesores son los que no asisten a las convocatorias que se formulan para capacitación. Los profesores no están modificando sus hábitos para incluir las nuevas tecnologías en su vida cotidiana y laboral de manera efectiva, por cual se verán afectados por la globalización y no se están preparando adecuadamente para el tipo de estudiantes que reciben y recibirán. La cobertura de la internet no es completa en el país y no todo hogar la posee, pero no hay tampoco la disposición de buscar alternativas para recibir la capacitación.

El reto del proyecto será of recer formación por internet y a distancia, de manera que los docentes vayan familiarizándose con nuevas tecnologías. Al igual que se analizó la situación de los docentes de primaria, esta actitud semejante en los prof esores debe enfrentarse como un gran desafío, que lejos de disminuir el estímulo para el uso de la tecnología debe fortalecer la decisión y la intervención de la UNA como motor de renovación y de actualización de la educación nacional.

Otra de las preguntas buscó medir el nivel de conocimiento que el público docente posee del proyecto Francés y Educación. Se constató que el proyecto era conocido únicamente por 35 docentes (19\% de los encuestados), pero prácticamente todos, 169 (un 95\%) manifestaron su deseo de recibir nuestros boletines y las informaciones pedagógicas que pudiéramos suministrarles, así como las comunicaciones respecto de los talleres que eventualmente se of recieran. PROFE considera que el primer paso para estimular el deseo de formación continua y el contacto con la UNA será iniciar un proceso de sistematización del envío de boletines e incrementar la interacción con los docentes. 
Los encuestados indicaron cuáles temas escogerían para eventuales talleres de capacitación. Al haber posibilidad de una respuesta múltiple, interpretamos, con base en el comportamiento de cada región y el interés mostrado por los docentes en los diversos temas, como elementos de predicción su interés en seguir realmente uno de los talleres. En todas las zonas urbanas (especialmente San José, Desamparados y Heredia), los docentes escogieron varios temas simultáneamente, lo cual podría interpretarse como un mayor y diversificado interés por las actividades de formación. En cambio, en zonas rurales hubo incluso temas que ni siquiera fueron seleccionados como opciones deseables. Dentro de este grupo los docentes muestran poco interés por la actividad literaria o por temas sobre nuevas tecnologías, ya que tanto las creación de cuentos y poemas, así como las aplicaciones de la computadora en el aula y la radio en clase, ocuparon los últimos lugares de preferencia en varios sectores, como Alajuela, San Carlos, Cartago y Puntarenas.

Todos los sectores expresan la necesidad de contar con técnicas y entrenamiento en la expresión oral, tanto para profesores como para estudiantes, ya que ambas opciones "arrasaron" con las preferencias de los encuestados. En este punto, vale aclarar que un solo taller no sería suficiente para carencias de tipo oral, sino que habría que emprenderse una acción mucho más sistemática y efectiva. La pregunta sería si los docentes realmente estarían dispuestos a perseverar en una serie de talleres de expresión oral. Extrañamente, los profesores no utilizan, tanto como podrían, la opción de la radio, que provee justamente un período apropiado para entrenamiento de la comprensión oral.

Este informe concreta los puntos en que la encuesta nacional les puede servir como referencia a las entidades relacionadas con la enseñanza del francés. De estas encuestas, PROFE ha obtenido también una lista de 125 contactos aproximadamente, que se sumaron a los 75 con que se contaba, de manera que se ha completado a la fecha un total de casi 200 personas que a lo largo no sólo de nuestro país, sino 
incluso de Centroaméricamérica, han tenido noticia de la labor de PROFE y se han interesado por recibir información. Así, un valioso banco de datos está en procesamiento y que servirá para la localización e identificación de los compañeros docentes de francés. PROFE está estableciendo las preferencias de formación y de recepción de información, de manera que se contará con una base de datos que registra los contactos por vía electrónica, por vía postal, por medio de fax, por llamada telefónica. Como mencionamos anteriormente, la división realizada por regiones nos permite escoger los talleres de mayor demanda cuando se planea alguna visita regional, de manera que puedan atenderse con mayor precisión las necesidades y expectativas de los colegas.

El comentario, discusión y las futuras acciones que resulten de la labor interinstitucional permitirán una mejor dirección en los esfuerzos de formación continua y de apoyo pedagógico que las diferentes instancias nacionales han emprendido desde sus perspectivas.

Entre los aspectos destacados, se confirma que la distribución de los profesores con mayor grado académico se da en la capital (76\% de graduados universitarios) y las zonas predominantemente urbanas — como Desamparados (84\%), Cartago (67\%) y Heredia (75\%)— para disminuir drásticamente en otros sectores como San Carlos $(0,9 \%$ tienen grado universitario contra $68 \%$ de aspirantes nombrados como docentes), Cañas, Limón, Aguirre y Turrialba (ninguna persona con grado universitario fue entrevistada en estas regiones). En los sectores urbanos con mayor concentración de graduados los porcentajes de interinazgo alcanzan el 40\%; por el contrario, en sectores alejados se ha otorgado propiedad incluso a aspirantes, dada la inopia existente. Asimismo, se encontró una marcada preferencia (70\% de los encuestados) por la formación presencial, seguida por la formación a distancia $(25 \%)$ y por un interés contradictorio en la formación virtual $(65 \%$ se muestran interesados pero sólo $0,8 \%$ se matricularían). Los resultados del estudio han sido divulgados y son analizados en conjunto con 
las entidades participantes en su realización para la toma de acciones pertinentes ${ }^{16}$.

Con las actuales orientaciones pedagógicas de nuestros días y las variantes en las directrices de evaluación, se ha hecho necesario proyectar la producción de materiales didácticos actualizados y acordes con los programas y las exigencias modernas. Actualmente se preparan materiales de lectura fundamentados en diferentes aproximaciones, tales como el constructivismo, la interculturalidad, la interactividad, el método ecléctico, etc.

La formación inicial es otro de estos campos: fue en el PROFE donde nació la colaboración de la UNA en el proyecto de francés de primaria del Ministerio de Educación Pública mediante el Programa de Lenguas Extranjeras para el Desarrollo (PROLED). Desde el MEP se trató de implementar un convenio de formación de docentes de francés en primaria, que no cuajó, principalmente por cambios de las autoridades del ministerio. La colaboración pedagógica y el apoyo en formación inicial para las maestras de primaria se continuaron; paralelamente en la Universidad Nacional se analizó lo sucedido con el convenio con el MEP, por lo cual apoyó la idea de continuar de manera interna la coordinación interfacultades entre la sección de Educación Básica del Centro de Investigación y Docencia en Educación (CIDE) y la Escuela de Literatura y Ciencias del Lenguaje mediante una comisión, con el fin de crear sendos Bachilleratos en la Enseñanza del Francés y del Inglés para primero y segundo ciclos. Así, además de velardurante tantos años por la actualización de los docentes de todas partes del país, también se ha preocupado por garantizar y proponer planes universitarios de formación docente.

Con un plan de estudios listo para ponerse en marcha, queda aún pendiente la producción de material específico para la enseñanza del francés a nivel preescolar y escolar, así como la investigación de la adquisición precoz de una lengua extranjera en nuestro medio particular;

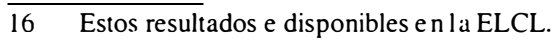


ambas iniciativas dieron origen a un nuevo proyecto en conjunto con el Centro de Investigación y Docencia en Educación (CIDE) para 2006, que posterga su puesta en marcha para 2007.

Frente al desafío de las nuevas tecnologías en la enseñanza de las lenguas extranjeras, se presenta una serie de retos importantes y urgentes en relación directa con los objetivos iniciales del proyecto. PROFE ha ido ampliando sus horizontes en los últimos años y la coyuntura histórica ha ido perfilando nuevas orientaciones en su labor.

La producción radiofónica fue el primero de estos esfuerzos, con la cobertura y el servicio a disposición de un numeroso público a lo largo y ancho del país. Actualmente, con el apoyo de la cooperación francesa, se realizan cuatro emisiones de 30 minutos por semana y Fréquence Ado se ha convertido en el 2005 en un nuevo proyecto, con autonomía en sus metas y productos.

En 2004, PROFE incluyó el uso de la consultoría y las comunicaciones con el profesorado, mediante correo electrónico, con una distribución mensual de boletines pedagógicos que ha derivado también en el diseño y la preparación de una página web en el sitio de la Facultad de Filosofía y Letras ${ }^{17}$. Gracias a estos medios, se contemplan diversos vínculos para profesores y estudiantes, así como la pronta posibilidad de talleres en línea. Los contactos que se han concretado incluyen profesores centroamericanos y se espera ampliarlos a otras latitudes, lo cual ofrece una perspectiva ya no sólo nacional sino regional, e incluso internacional de la labor del proyecto y de la UNA. La inclusión de Internet permite poner a disposición de un amplio público los productos del proyecto que mantienen vigencia y que han sido ya digitalizados, como las primeras emisiones radiofónicas.

$17<$ <www.elcl.una.ac.cr/extension/PROFE/Principale.htm>; Además, se le asignó al proyecto una dirección de correo electrónico: <pfe@ una.ac.cr>: y cuenta con una lista de difusión para los 200 contactos: < rances@listserv.una.ac.cr $>$. 


\section{Viabilidad y factibilidad académica dentro del contexto actual}

Costa Rica vive una situación social y política que obliga la presencia de la Universidad Nacional en pro del humanismo, la participación y la solidaridad. Esta situación se relaciona con un contexto que ha modificado el panorama laboral y económico de muchos países. Una de las funciones de los formadores universitarios es permitir al estudiante el contacto con su realidad laboral y la responsabilidad de estar un paso delante de los cambios a los que deberá enfrentarse. Si a esta responsabilidad sumamos la necesidad de incluir y no de excluir PROFE se confirma como una iniciativa valiosa y solidaria, humanista y progresista.

En términos generales, se les reconoce su importancia a las lenguas extranjeras como instrumentos de comunicación y de intercambio, tanto en la comunicación cotidiana como en las transacciones políticas y comerciales. Esto se ha visto favorecido por los avances tecnológicos en la comunicación. El ejemplo de la diversificación linguística de la Comunidad Europea confirma la necesidad del dominio, ya no de una lengua extranjera, sino de aquellas que sea posible aprender y utilizar. Actualmente se deben conocer varios idiomas para establecer y mantener negocios e intercambios con otras naciones. En Costa Rica, el Ministerio de Educación Pública ha incrementado modalidades educativas que incluyen el francés y ha ampliado la cantidad de escuelas que lo tienen como lengua extranjera. Esta realidad educativa ha multiplicado las necesidades de formación inicial y de la continua, especialmente acentuadas en las zonas alejadas, donde no se cuenta con of erta universitaria. Si el profesorado en activo cuenta con opciones de capacitación, serán la niñez y la juventud las que se verán directamente beneficiadas. Además del reto que presenta la planificación e investigación en las áreas que se han derivado de la propuesta inicial, académicamente existe una necesidad nacional y una inquietud generalizada en los profesores de francés frente a la globalización, que impulsa aún más a los profesores como formadores de formadores, incursionar en otras posibilidades pedagógicas para beneficio del país. 


\section{Apéndice 1. Encuesta aplicada a la muestra de profesores de se- cundaria (y de primaria, con pequeñas modificaciones) para el diagnóstico realizado en el período 2004-2005}

\section{UNIVERSIDAD NACIONAL \\ ESCUELA DE LITERATURA Y \\ CIENCIAS DEL LENGUAJE \\ SECCIÓN DE FRANCÉS}

EnCUesta PARa PROFESORES de SECUNDARIa

Estimado colega: Agradecemos de antemano su colaboración. La información que usted nos brinde permitirá crear

oportunidades de estudio y mejoramiento profesional para usted, por lo que le rogamos ser lo más objetivo(a)

posible. Toda esta información se manejará con estricta confidencialidad.

1. Nombre completo:

2. Institución donde labora: Tel:

3. Niveles a cargo $\square$ 7mo $\square$ 8vo $\square$ 9no $\square$ 10mo $\square$ 11mo

4. Grupo profesional asignado:__ Situación laboral: $\square$ propiedad $\square$ interino 5. ¿Dónde obtuvo su conocimiento de la lengua francesa?

$\square$ durante una estadía en Francia francófono

$\square$ por estudios en el Liceo Franco

$\square$ estudios en otro país (¿cuál?

$\square$ durante una estadía en otro país $\square$ otro: explique

6. ¿Ha realizado estudios? $\square$ no $\square$ sí (¿dónde?) $\square$ UNA (presencial)

$\square$ Alianza Francesa $\square$ UCR (presencial) $\square$ Convenio UCR-UNED

Último nivel aprobado: ¿Estudia actualmente aún? $\square$ $\square$ sí $\square$ no 7. ¿Qué modalidad de estudio prefiere? $\square$ Presencial $\square$ A distancia $\square$ virtual 8. ¿Cuál de los siguientes horarios escogería usted para recibir talleres o capacitación?

$\mathrm{a} \square$ Viernes matutino (entre 7 y 12 hrs.) $\square$ Viernes tarde (1 a 6)

c $\square$ Lunes a viernes nocturno (después de las $5 \mathrm{pm}$ ) d $\square$ Sábado en la mañana 9. ¿Si la UNA ofrece talleres de capacitación y formación continua por Internet usted se matricularía?

$\square$ sí $\square$ no ¿por qué? 
10. Si la UNA entrega certificados de participación por cada taller que le sirvan para subir puntos de carrera profesional, se interesaría usted en los talleres

$\square$ presenciales $\square$ virtuales $\square$ ambos

11. ¿Conoce usted de proyecto Francés y Educación de la UNA? $\square$ sí $\square$ no

12. ¿Le interesaría recibir los boletines pedagógicos del proyecto? $\square$ sí $\square$ no

13. ¿Cuál medio considera más efectivo para recibir la información?

$\square$ enviarle un correo electrónico a $\square$ enviarle un fax al colegio $\square$ llamar a su colegio para invitar a los talleres $\square$ enviarle la información por vía postal $\square$ otro:

14. ¿Qué temas de formación escogería para los talleres?

$\square$ expresión oral para el profesor

$\square$ cómo trabajar la literatura en clase secundaria $\square$ ayuda técnica para las pruebas nacionales

$\square$ elaboración de exámenes de aula

$\square$ corrección fonética

aula $\square$ adecuaciones curriculares en francés

$\square$ la radio en la clase de francés

$\square$ estilos de aprendizaje y enseñanza

$\square$ elaboración de materiales creativos

$\square$ otros ¿cuáles?

Por favor; si desea agregar comentarios, preguntas o sugerencias, escriba al dorso de esta hoja, nos interesa mucho su opinión y las necesidades particulares de su región de trabajo.

Para mayor información: proyectoprofe@ hotmail.com. 


\section{Apéndice 2. Material utilizado por Róger Retana en una de las actividades realizadas durante los talleres de 2004 en las zonas rurales $^{*}$}

\section{GALICISMOS EN EL ESPAÑOL}

Palabras de origen francés empleadas en español. Puede variar la ortografía o la pronunciación.

¿De qué lengua son originarias las siguientes palabras? I= Italiano, In=Inglés, $\mathrm{A}=$ árabe, $\mathrm{N}=$ Nahúatl

\begin{tabular}{|l|l|l|l|l|l|l|l|l|}
\hline piano & azúcar & chocolate & cassette & buqué & disquette & garage & web & UHT \\
\hline & & & & & & & & \\
\hline
\end{tabular}

Las siguientes palabras son galicismos. Clasifíquelas por áreas temáticas.

\begin{tabular}{|l|l|l|l|l|l|l|l|l|l|}
\hline & cuisine & sport & spectacle & autres & & cuisine & sport & spectacle & autres \\
\hline chifonnier & & & & & begonia & & & & \\
\hline disquette & & & & & bidé & & & & \\
\hline crepa & & & & & brochure & & & & \\
\hline buffet & & & & & cassette & & & & \\
\hline matinée & & & & & champinón & & & & \\
\hline ensemble & & & & & chasis & & & & \\
\hline repris & & & & & chic & & & & \\
\hline croissant & & & & & debut & & & & \\
\hline chef & & & & & flambé & & & & \\
\hline carné & & & & & gato & & & & \\
\hline corsé & & & & & mousse & & & & \\
\hline beige & & & & & negligé & & & & \\
\hline gratinado & & & & & papel maché & & & & \\
\hline bobina & & & & & puré & & & & \\
\hline surmenage & & & & & savoir-faire & & & & \\
\hline pasteurización & & & & & vedette & & & & \\
\hline
\end{tabular}

* Fuentes: <http://www.idiomaydeporte.com/pages/base/htm>. Henriette Walter, Le français dans tous les sens (Paris : Le Livre du Poche, 1996): Jorge Guasch, Galicismos, aceptados, aceptables y vitandos. (Buenos Aires: Kapelusz, 1951). 
Construcción de palabras: pomme de terre, poule-coq d'Inde (dinde).

Otras palabras : bebé, boutique, bricolage, bulevar, chalet, chofer, foie gras, nécessaire (de toillette), restaurante.

El español en francés: chayote, corrida, fiesta, chocolate, tomate, plátano, guineo, banano.

SOPA DE LETRAS: GALICISMOS

\begin{tabular}{|c|c|c|c|c|c|c|c|c|c|}
\hline B & R & O & C & H & U & R & E & M & F \\
\hline I & W & T & J & K & H & E & C & A & L \\
\hline D & M & C & X & D & T & P & E & C & A \\
\hline E & O & E & L & I & A & R & W & H & M \\
\hline T & E & R & N & C & H & I & C & E & B \\
\hline G & A & T & O & U & A & S & Z & M & E \\
\hline C & C & A & S & S & E & T & T & E & K \\
\hline S & U & R & M & E & N & A & G & E & X \\
\hline C & H & A & M & P & I & G & N & O & N \\
\hline E & T & U & O & M & O & U & S & S & E \\
\hline
\end{tabular}

